\title{
Evaluation of Mean Thrombocyte Volumes in Asthma Patients During Acute Exacerbations and Stable Periods
}

\author{
Uysal Dolap ${ }^{1}$, Tuncer Tuğ ${ }^{2}$, Suat Konuk ${ }^{2 *}$ \\ ${ }^{1}$ Şırnak State Hospital, Şırnak, Turkey \\ ${ }^{2}$ Abant İzzet Baysal Univercity, Medicine Faculty, Bolu, Turkey
}

Corresponding Author: Suat Konuk, Abant İzzet Baysal Univercity, Medicine Faculty, Bolu, Turkey, Tel: 90 507341 0126; E-mail: suatkonukk@windowslive.com

Received: 10 January 2018; Accepted: 22 January 2018; Published: 25 January 2018

\begin{abstract}
Objective: Asthma is a heterogeneous disease characterized by chronic airway inflammation. Mean Platelet Volume (MPV) is one of the markers of platelet activation. The disease activity has been shown to have an important role as a marker for the efficacy of anti-inflammatory therapy and inflammation. The aim of this study was to investigate the relationship between disease activity and mean platelet volume and to determine the mean platelet volume (MPV) and CRP values during asthma exacerbations and stable periods.
\end{abstract}

Methods: In this study, data from 50 patients with asthma exacerbations, 50 stable asthmatic patients and 40 healthy subjects were evaluated retrospectively.

Results: Even though the MPV values of patients with an asthma exacerbation was lower $(9.2 \pm 0.9 \mathrm{fL})$ than those of stable asthmatic patients $(9.6 \pm 1.3 \mathrm{fL})$, we did not find statistical differences between the groups in terms of MPV results $(P=0.374)$. Leukocyte, neutrophil counts and percentages and CRP levels were significantly higher in patients with an exacerbation than in stable asthmatic and control groups $(p=0.000)$. There was no difference in , neutrophil counts and percentages and CRP levels between stable asthmatic patients and healthy controls ( $\mathrm{p}>0.05)$.

Conclusion: In conclusion, there was no statistically significant relationship between the MPV values and disease activity in asthmatic patients. We believe that prospective studies involving a larger number of patients are needed to determine the place of MPV in asthmatic patients, since the number of patients and controls in our study was low. 
Keywords: Asthma; Mean Platelet Volume; Thrombocyte volume; Chronic airway inflammation

\section{Introduction}

Asthma is a heterogeneous disease generally characterized by chronic airway inflammation. It is a chronic inflammatory disease of the airways that affects $1-18 \%$ of the population in different countries. It is characterized by wheezing, shortness of breath, tightness in the chest, cough, and variable limitation of expiratory airflow [1]. Asthma is an inflammatory disease of the airways and includes many inflammatory cells and mediators that result in the characteristic pathophysiological changes. Inflammation is also known to be strongly associated with bronchial hyperresponsiveness and asthma symptoms [2]. Although symptoms are episodic, airway inflammation in asthma is persistent. Inflammation affects all airways, including the nose and upper airways in many patients, but the physiological effects are most pronounced in the medium-sized bronchi [1]. Mean platelet volume (MPV) is one of the routine parameters in whole blood count tests and indicates the mean volume of circulating platelets. MPV is one of the indicators of platelet activation. Circulating platelets vary in size and function. Large platelets are younger and more active than small platelets and have greater thrombogenicity $[3,4]$.

In many chronic inflammatory diseases, the critical role of mean platelet volume (MPV) as a measure of disease activity, efficacy of anti-inflammatory therapy and inflammation was shown. In inflammatory diseases, the size of thrombocytes is affected by the severity of systemic inflammation. The mean platelet volume (MPV) decreases with antiinflammatory therapy [5]. C-reactive protein (CRP) is one of the positive acute phase proteins showing inflammation and is widely used as a sensitive qualitative/quantitative test showing tissue damage and inflammation in routine laboratory tests [6].

The purpose of this study is to determine mean platelet volume (MPV) and CRP (C-Reactive Protein) values during asthma exacerbations and stable periods, to investigate the relationship between disease activity and mean platelet volume and to compare mean platelet volume (MPV) values of asthmatic patients and healthy subjects.

\section{Materials and methods}

In this study, data from 50 asthma exacerbations, 50 stable asthmatic patients who were followed up at AİBÜ Medical Faculty Hospital Emergency Department, Chest Diseases Outpatient Clinic and Services between April 2013 and April 2014 and data from 40 healthy subjects who were admitted to Chest Diseases Policlinic and who had no pathological findings were evaluated retrospectively. Age, gender, hemogram, and CRP results were recorded in all cases. Patients who had any systemic inflammatory disease, severe anemia or hematologic disease, malignancy, chronic liver or kidney disease, and who were using systemic corticosteroids, antiinflammatory or anticoagulant medications were excluded from the study.

\subsection{Statistical analysis}

For statistical analysis of the data, "SPSS (Statistical Package for Social Sciences) for Windows 16.0" package 
program was used. After entry of the patients' data into the SPSS program, continuous variables were expressed as mean \pm standard deviation and categorical variables as percent $(\%)$ in subgroup comparisons. The t-test was used to compare the numerical parameters among the groups, the Many-White test was used for the non-parametric tests, and the chi-square test was used for the comparison of categorical variables. Bivariate Pearson test was used for correlation tests. A p value of $<0.05$ was considered statistically significant in all tests.

\section{Results}

This study included 50 stable asthmatic patients [39 Women (W), 11 Men (M); (mean age: $44.5 \pm 17.2$ years), 50 asthmatic patients with exacerbations $(38 \mathrm{~W}, 12 \mathrm{M}$, mean age: $47.1 \pm 15.4$ years) and 40 healthy controls (31 W, 9 M, mean age: $45.8 \pm 4.5$ years). There was no statistically significant difference between the three groups in terms of gender and age $(\mathrm{p}>0.05)$.

Hematological parameters (including MPV) and CRP values of the three groups were compared. No statistically significant difference was found between the asthmatic patients, patients with exacerbations and the control group (p $=0.374)$. There was no statistically significant difference between the three groups in other hematological parameters: hemoglobin, hematocrit, PDW and eosinophil percentage ( $\mathrm{p}>0.05)$. Leukocyte, neutrophil counts and percentages and CRP levels were significantly higher in patients with an exacerbation than in stable asthmatic and control groups $(\mathrm{p}=0.000)$ (Table 1$)$. There was no difference in, neutrophil counts and percentages and CRP levels between stable asthmatic patients and healthy controls $(\mathrm{p}>0.05)$. Hemoglobin $(\mathrm{Hb})(\mathrm{p}=0.002)$ and hematocrit $(\mathrm{Hct})$ $(\mathrm{p}=0.006)$ values were found to be lower in women. There was no gender based difference in CRP, MPV and other hematological parameters in asthmatic patients with exacerbations. There was no correlation between age and OTH in this group $(\mathrm{p}>0.05, \mathrm{r}=0.001) \mathrm{Hb}(\mathrm{p}=0.000)$ and Hct values $(\mathrm{p}=0.000)$ were lower in women the stable asthmatic group. In this group, there was no gender based difference in terms of CRP, MPV and other hematological parameters $(\mathrm{p}>0.05)$. There was no correlation between age and OTH $(\mathrm{p}>0.05, \mathrm{r}=0.113)$.

\begin{tabular}{|c|c|c|c|c|}
\hline & Exacerbation & Stable & Control & p value \\
\hline Sex n, (\%) & & & & \\
\hline Woman & $38 /(76)$ & $39 /(78)$ & $31 /(77.5)$ & \\
\hline Mean age \pm SD & $47.1 \pm 15.4$ & $44.5 \pm 17.2$ & $45.8 \pm 14.0$ & 0.726 \\
\hline CRP (mg/L) & $23.5 \pm 23.9$ & $1.9 \pm 1.2$ & $1.9 \pm 1.2$ & $<\mathbf{0 . 0 0 1}$ \\
\hline Leucocyte (K/Ul) & $9650 \pm 3281$ & $6700 \pm 1475$ & $6960 \pm 1416$ & $<\mathbf{0 . 0 0 1}$ \\
\hline Neutrophile ( 10^9/L) & $6890 \pm 2810$ & $4130 \pm 1164$ & $4230 \pm 1050$ & $<\mathbf{0 . 0 0 1}$ \\
\hline Neutrophile (\%) & $71.0 \pm 9.0$ & $60.9 \pm 6.0$ & $60.7 \pm 7.7$ & $<\mathbf{0 . 0 0 1}$ \\
\hline Hb (gr/dl) & $13,4 \pm 1,5$ & $13,9 \pm 1,4$ & $13,8 \pm 1,3$ & 0.198 \\
\hline Hct (\%) & $40.7 \pm 4.0$ & $41.6 \pm 3.6$ & $41.0 \pm 3.7$ & 0.572 \\
\hline
\end{tabular}




\begin{tabular}{|c|c|c|c|c|}
\hline Thrombocyte & $301820 \pm 72373$ & $283420 \pm 86819$ & $27878 \pm 49934$ & 0.285 \\
\hline Eosinophil percentage & $2.3 \pm 2.0$ & $2.2 \pm 1.3$ & $2.1 \pm 1.4$ & 0.720 \\
\hline MPV & $9,2 \pm 0,9$ & $9.6 \pm 1.3$ & $9.3 \pm 1.0$ & 0.374 \\
\hline PDW & $15.6 \pm 1.3$ & $15.0 \pm 1.5$ & $14.8 \pm 1.6$ & 0.244 \\
\hline
\end{tabular}

Table 1: Demographic characteristics stable, exacerbation and control groups and the comparison of CRP, MPV and other hematological parameters.

\section{Discussion}

In our study, we did not find any statistical difference between patients with asthma exacerbations, stable asthmatic patients and healthy subjects in terms of MPV values. We found CRP values, leukocyte, neutrophil counts, and neutrophil counts were higher in exacerbation group than those in the stable asthmatic and control groups.

Mean platelet volume (MPV) is a parameter that is routinely measured in full blood count devices. Mean platelet volume (MPV) is a marker of active platelets. Recent studies have claimed that the levels of MPV values drop due to the consumption of active or large platelets in severe inflammatory conditions and that this can be reversed by anti-inflammatory treatments [5]. In one study, children with active nephrotic syndrome had a lower MPV than controls [6]. In another study, MPV values were found to be lower in patients with ulcerative colitis compared to the control group, and in patients with active ulcerative colitis, the levels of MPV were lower than those of non-active ulcerative colitis [7]. In separate study, patients with rheumatoid arthritis (RA) and ankylosing spondylitis were found to have lower values of MPV compared with the control group and MPV values increased after treatment [8]. Another study in patients with RA showed an increase in MPV values during anti-TNF therapy [5].

In asthmatic patients, only a few studies have examined the MPV values during an exacerbation. In a recently published study, Sun WX et al.[9] compares the values of OTH, RDW, CRP and leukocyte in 85 stable asthmatic patients, 85 asthmatic patients with an exacerbation and 85 control patients. MPV values were lower compared to the control group $(\mathrm{p}<0.001)$ and the stable group $(\mathrm{p}=0.003)$. Patients with an exacerbation $(9.8 \pm 0.8 \mathrm{fl})$ had lower values of MPV compared to those with stable asthma $(10.3 \pm 1.1 \mathrm{fl})(\mathrm{p}=0.008)$. In a study by Tuncel T et al. [10] of 100 asthmatic children did not find a difference in MPV values between the control, stable and exacerbation groups.

The role of platelets in asthma has been well documented in recent years [11]. In murine models, leukocyte infiltration and airway remodeling cannot occur without the involvement of platelets [12]. Systemic inflammation increases in asthma attacks. Proinflammatory cytokines such as IL-6, TNF-alpha and CRP increase during asthma exacerbations [13,14]. Platelets act as companions in the process of extravasation of leukocytes into bronchopulmonary airways from pulmonary microcirculation and in chemotaxis stimulation [15]. Aggregation of 
platelets is accelerated during hypoxia and/or hypercapnia. Platelet activating factor in asthma can aggravate and activate platelets and can have substantial proinflammatory effects [16]. In our study, we found that patients with an exacerbation had lower values of MPV compared to those with stable asthma, but this result was not statistically significant. Again, in our study, the MPV values in the control group were the same as those in patients with an exacerbation. MPV vales were lower in stable asthmatic patients. The control group consisted of healthy people. Yet these were people who came to the hospital for various reasons. Therefore, more careful selection of the control group in new studies may be important in terms of achieving more reliable results. Furthermore, studies involving more asthmatic patients may lead to clearer results in this regard. In addition, in the same patient group in asthmatic adults, the detection of MPV values in exacerbations and stable periods may contribute to the literature.

In conclusion, we did not find a statistical difference in the MPV values between stable patients, patients with an exacerbation and a healthy control group. The fact that the MPV values were lower in the exacerbation group than the stable group was consistent with the study results. This difference in our study was not statistically significant and our patient population was small. The lack of publications in this area limits our ability to comment further. More studies involving more patients will lead to clearer results.

\section{Acknowledgment}

None

\section{Conflict of Interest}

The author declares that he has no conflict of interest

\section{References}

1. Bateman ED, Hurd SS, Barnes PJ et al. Global strategy for asthma management and prevention: GINA executive summary. The European respiratory journal 31 (2008): 143-178.

2. Devereux G, Seaton A. Diet as a risk factor for atopy and asthma. J Allergy Clin Immunol 115 (2005): 1109-1117.

3. Briggs C. Quality counts: new parameters in blood cell counting. International journal of laboratory hematology 31 (2009):277-297.

4. van der Loo B, Martin JF. Megakaryocytes and platelets in vascular disease. Baillieres Clin Haematol 10 (1997): 109-123.

5. Gasparyan AY, Ayvazyan L, Mikhailidis DP, Kitas GD. Mean platelet volume: a link between thrombosis and inflammation? Current pharmaceutical design 17 (2011): 47-58.

6. Gulleroglu K, Yazar B, Sakalli H, Ozdemir H, Baskin E. Clinical importance of mean platelet volume in children with nephrotic syndrome. Renal failure 36 (2014): 663-665.

7. Yuksel O, Helvaci K, Basar O et al. An overlooked indicator of disease activity in ulcerative colitis: mean platelet volume. Platelets 20 (2009): 277-281. 
8. Kisacik B, Tufan A, Kalyoncu U et al. Mean platelet volume (MPV) as an inflammatory marker in ankylosing spondylitis and rheumatoid arthritis. Joint, bone, spine : revue du rhumatisme 75 (2008): 291294.

9. Sun WX, Zhang JR, Cao ZG, Li Y, Wang RT. A decreased mean platelet volume is associated with stable and exacerbated asthma. Respiration; international review of thoracic diseases 88 (2014): 31-37.

10. Tuncel T, Uysal P, Hocaoglu AB, Erge DO, Karaman O, Uzuner N. Change of mean platelet volume values in asthmatic children as an inflammatory marker. Allergologia et immunopathologia 40 (2012): 104107.

11. Pitchford SC. Novel uses for anti-platelet agents as anti-inflammatory drugs. British journal of pharmacology 152 (2007): 987-1002.

12. Pitchford SC, Riffo-Vasquez Y, Sousa A et al. Platelets are necessary for airway wall remodeling in a murine model of chronic allergic inflammation. Blood 103 (2004): 639-647.

13. Yokoyama A, Kohno N, Fujino S et al. Circulating interleukin-6 levels in patients with bronchial asthma. American journal of respiratory and critical care medicine 151 (1995): 1354-1358.

14. Deraz TE, Kamel TB, El-Kerdany TA, El-Ghazoly HM. High-sensitivity C reactive protein as a biomarker for grading of childhood asthma in relation to clinical classification, induced sputum cellularity, and spirometry. Pediatr Pulmonol 47 (2012): 220-225.

15. Kornerup KN, Page CP. The role of platelets in the pathophysiology of asthma. Platelets 18 (2007): 319 328.

16. Kasperska-Zajac A, Brzoza Z, Rogala B. Platelet activating factor as a mediator and therapeutic approach in bronchial asthma. Inflammation 31 (2008): 112-120.

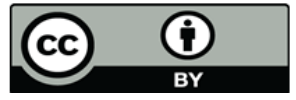

This article is an open access article distributed under the terms and conditions of the Creative Commons Attribution (CC-BY) license 4.0 